\title{
II contributo dei fertilizzanti all'effetto serra nella filiera vitivinicola
}

\author{
Claudio Pattara ${ }^{1}$, Leo Giannantonio ${ }^{2}$, Vincenzo Lorito ${ }^{2}$, Roberta Vinciguerra ${ }^{2}$, Marco Piscicelli $^{2}$, e Angelo Cichelli ${ }^{1}$ \\ ${ }^{1}$ DEc. Dipartimento di Economia, Università G. d'Annunzio Pescara, Italia \\ 2 Valagro SPA, Atessa, Italia
}

\begin{abstract}
Sintesi. La filiera vitivinicola è una attiva produttiva che non può essere considerata esente impatti ambientali correlati. Come è noto nel ciclo di vita del vino la fase più impattante è quella agricola. Ciò a causa dell'utilizzo dei combustibili fossili per i macchinari agricoli, dei prodotti fitosanitari per la protezione della vite e per l'uso di pratiche di fertilizzazione per il mantenimento di alte rese per ettaro. Un aspetto importante è quello legato alle emissioni di gas ad effetto serra (GHG) in un'ottica di ciclo di vita del prodotto. Le pratiche di fertilizzazione del terreno in ambito viticolo prevedono la somministrazione di azoto, fosforo e potassio e altri microelementi (Mg, $\mathrm{Fe}, \mathrm{Bo}, \mathrm{Zn}, \mathrm{Cu}$ ), tali da garantire livelli produttivi adeguati alle necessità della azienda agricola. Tale quantitativo varia in base alle caratteristiche del terreno, come ad esempio il contenuto in sostanza organica. In questo studio, sulla base delle pratiche frequenti a livello regionale (Abruzzo), è stata comparata la possibile gestione di un vigneto (Montepulciano) secondo due principali modalità di concimazione. La prima secondo le pratiche del disciplinare della Regione Abruzzo, e nella seconda attraverso una gestione standard e con un apporto di $600 \mathrm{Kg}$ di fertilizzante NPK. Dall' analisi dei dati è emerso che la pratica della fertilizzazione rappresenta una componente molto importante in termini di emissioni di GHG. Si nota che le due tecniche operative mostrano che il solo utilizzo di prodotti NPK genera un quantitativo di emissioni superiore di circa il 30\% alla procedura con NPK e trattamenti fogliari. Questo risultato è da attribuire principalmente al ridotto utilizzo di concimi NPK e in parte al fatto che i quantitativi di prodotti fogliari utilizzati (2-31/ha per singolo trattamento) sono di gran lunga inferiori rispetto ai concimi granulari standard di tipo NPK.
\end{abstract}

\section{Introduzione}

L'aumento della concentrazione di gas ad effetto serra (GHG) è considerata la causa principale dei cambiamenti climatici mondiali [25]. Fra questi gas la $\mathrm{CO}_{2}$ è aumentata da $280 \mathrm{ppm}$ nel 1975 , a più di $380 \mathrm{ppm}$ nel 2010 e la sua crescita è costante $(+1,5 \mathrm{ppm} / \mathrm{anno})$ [25]. Allo stesso modo anche gli altri GHG hanno raggiunto concentrazioni elevate; ad esempio la concentrazione atmosferica di $\mathrm{CH}_{4}$ è passata da $722 \mathrm{ppb}($ nel 1750) a 1800ppb (nel 2011) con una crescita del $150 \%$, allo stesso modo la $\mathrm{N}_{2} \mathrm{O}$ è anno passata da $271 \mathrm{ppb}$ a $324,2 \mathrm{ppb}$ nello stesso intervallo di anni [25].

Le principali fonti di emissione di GHG derivano dai combustibili fossili che, nel corso del $20^{\circ}$ secolo, sono stati utilizzati in maniera drastica sia per attività energetiche che per quelle manifatturiere [20]. Il Protocollo di Kyoto prevede per i paesi firmatari l'obbligo di operare una riduzione delle emissioni di questi gas entro il 2020. Tuttavia esso non è ancora riuscito a raggiungere $\mathrm{i}$ traguardi attesi [23-25]. Le emissioni di GHG non sono l'unica problematica a cui fa riferimento il protocollo di Kyoto, infatti l'effetto serra è determinato anche dalla riduzione dei bacini di stoccaggio del carbonio (es: foreste) la cui superficie è andata diminuendo costantemente nel corso di tutto il secolo [27].

Questa problematica è stata internalizzata anche dall'Unione Europea (EU) [6-16] che ha considerato la lotta ai cambiamenti climatici come una delle priorità di azione in tutti i settori (energia, trasporti, agricoltura e zootecnia, etc.) ed ha inserito specifiche misure per la riduzione delle emissioni di GHG all'interno dei suoi piani di azione.

In considerazione di quanto premesso occorre soffermarsi sulle attività che generano GHG e nello specifico le attività legate ai settori agroindustriali rappresentano una componente in rapida crescita [37], è stato stimato infatti che circa 19-29\% delle emissioni di GHG è da attribuire al comparto agricolo e zootecnico. Circa 1'80-86\% di queste sono causate dalla produzione di prodotti agricoli $[7,8]$, mentre la restante parte può essere attribuita alla estrazione delle materie prime inserite nei processi produttivi (principalmente fertilizzanti) e nei processi di post-produzione.

In questo contesto [19] l'OIV ha pubblicato nel 2011 [30] il protocollo per il conteggio dei gas serra all'interno delle attività vitivinicole; esso presenta due possibili alternative per i calcoli dei GHG emessi. È previsto il conteggio a livello aziendale e a livello di prodotto. In questi mesi, a cura del gruppo di lavoro ad hoc, si stanno sviluppando gli allegati del protocollo che sono destinati a fornire un supporto tecnico robusto (coefficienti di emissione, linee guida di applicazione, metodologia di calcolo) per tutti gli stakeholder che decidono di implementare il protocollo all'interno della propria struttura.

Esistono tuttavia forme di gestione agricola che presentano forti caratteri di sostenibilità e che hanno

This is an Open Access article distributed under the terms of the Creative Commons Attribution License 4.0, which permits unrestricted use, distribution, and reproduction in any medium, provided the original work is properly cited. 
la possibilità di contribuire alla riduzione dei GHG in atmosfera attraverso la fissazione del carbonio nella biomassa vegetale e nel suolo e, in parte, promuovendo attività contraddistinte da basse emissioni [27-42]. In questo senso alle colture agrarie, ancora oggi, viene attribuita una semplice valenza produttiva a discapito di quella ambientale che è riconosciuta a livello globale agli ecosistemi forestali.

Tra tali colture è possibile includere vitivinicoltura che, nelle sue forme varie forme di allevamento può avere una funzione ambientale attraverso la stabilizzazione (comunemente chiamato stoccaggio) della $\mathrm{CO}_{2}$ atmosferica nelle proprie strutture vegetali e nel suolo [36-42]. Il carbonio atmosferico è l'elemento di base di tutta la struttura della vite e la crescita delle stesse si ritiene possa stoccare elevate quantità di carbonio atmosferico sia nelle strutture lignee che nel suolo. Sebbene la multifunzionalità della vitivinicoltura (come biodiversità e come stoccaggio di carbonio) sia ormai oggi largamente riconosciuta a livello internazionale, per essa non sono ancora presenti dati certi circa le quantità di carbonio che riesce a immobilizzare nelle strutture e nel terreno. In considerazione del carattere fortemente sperimentale del presente lavoro (e della necessità di dati pluriennali per le stime di carbonio nelle strutture lignee e nel suolo) è stato scelto di non includere considerazioni sullo stoccaggio di carbonio nel suolo e nelle piante. Sulla base di quanto premesso e nell'ottica di poter fornire un fattivo contributo sia alla riduzione dei GHG in ambito vitivinicolo che allo studio dei metodi di applicazione del Protocollo OIV, nel presente studio vengono riportati i dati relativi alla comparazione di due tecniche di conduzione del vigneto nella realtà Abruzzese.

\section{Materiali e metodi}

\subsection{Premessa metodologica}

Il settore vitivinicolo viene analizzato tramite la metodologia LCA già da numerosi anni $[3-5,28,29]$ ed ha contribuito in maniera significativa al miglioramento dell'informazione relativa agli impatti ambientali correlati ai sistemi produttivi permettendo di essere al contempo un efficace strumento su cui basare scelte di carattere operativo anche a livello di singola azienda agricola [33]. Le applicazioni di LCA al settore del vino sono numerose [33-36] e altrettanto numerose sono le applicazioni di Carbon Footprint. Il settore vitivinicolo è ad oggi un settore produttivo globale in cui si confrontano costantemente tutti i produttori da tutte le nazioni su aspetti sia qualitativi che ambientali. Per questo motivo l'impronta di carbonio è divenuta uno strumento chiave (anche per fini di marketing) su cui le più importanti aziende vitivinicole cercano di competere. La costante riduzione delle emissioni di GHG legate alla manifattura del vino è l'obiettivo verso cui moltissime aziende europee (italiane, francesi e spagnole principalmente) si stanno muovendo.

\subsection{Caso studio}

In Abruzzo la coltivazione della vite da vino interessa una superficie di 36.000 ettari per una produzione annua di 3,8 milioni di ettolitri $[33,34]$. La produzione di vini a
Tabella 1. Operazioni colturali.

\begin{tabular}{|l|l|l|}
\hline & Caso 1 & \multicolumn{1}{|c|}{ Caso 2 } \\
\hline $\begin{array}{l}\mathrm{N}^{\circ} \\
\text { Trattamenti } \\
\text { fitosanitari }\end{array}$ & \multicolumn{2}{|c|}{10} \\
\hline Agrofarmaci & \multicolumn{2}{|c|}{$\begin{array}{l}\text { Adatti alla lotta delle principali fitopatolo- } \\
\text { gie del Montepulciano presenti in Abruzzo } \\
\text { (peronospora, oidio, tignola, botrite) }\end{array}$} \\
\hline $\begin{array}{l}\text { Lavorazioni } \\
\text { meccaniche } \\
\text { del terreno }\end{array}$ & $\begin{array}{l}\text { 2 passaggi con } \\
\text { trinciasarmenti, } \\
\text { 1 vangatura }\end{array}$ & $\begin{array}{c}\text { 1 ripper } \\
1 \text { vangatura } \\
1 \text { trinciatura }\end{array}$ \\
\hline
\end{tabular}

denominazione di origine raggiunge il milione di ettolitri, oltre 800 mila dei quali sono di Montepulciano d'Abruzzo, 192 mila Trebbiano d'Abruzzo e 4 mila ciascuno per la DOC Controguerra e per la DOCG Montepulciano d'Abruzzo "Colline Teramane" [9].

Nei diversi areali di produzione, essa si caratterizza per un elevato grado d'integrazione tra la fase produttiva e quella della trasformazione fino ad assumere un ruolo strategico per il locale sistema socio-economico. La forma di allevamento maggiormente diffusa in Abruzzo è la "Pergola Abruzzese" che rappresenta oltre 1'80\% del vigneto regionale mentre nei nuovi impianti e reimpianti prevale nella maggior parte dei casi la forma a filare (cordone speronato, cordone libero). I tre quarti della produzione complessiva di vino in Abruzzo provengono da 40 cantine cooperative ( 30 delle quali operanti in provincia di Chieti) che unitamente a quelle private compongono un quadro di 160 aziende di trasformazione, 120 delle quali imbottigliano con propria etichetta.

Sulla base delle caratteristiche standard di conduzione dei vigneti di Montepulciano di Abruzzo DOC, e sulla base del disciplinare produttivo dello stesso è stata condotta una sperimentazione teorica. Sono stati considerati due areali produttivi di Montepulciano d'Abruzzo in territori della provincia di Chieti sui quali sono state applicate due tecniche di conduzione dei vigneti differenti. Per semplicità è stato ipotizzato che: terreni, condizioni climatiche, esposizione, precipitazioni, età del vigneto fossero uguali per entrambe le parcelle sottoposte alla sperimentazione. Per quanto riguarda il metodo di conduzione nella tabella sottostante vengono riportate le condizioni ipotizzate.

Per quanto riguarda i trattamenti fitosanitari è stato ipotizzato un quantitativo di $10 \mathrm{~m}^{3} / \mathrm{ha}$ di acqua per il processo di irrorazione, e un consumo di 10 litri di gasolio (diesel) per ogni trattamento (sono stati inclusi i possibili trasferimenti da e verso i terreni). I processi utilizzati sul software di LCA hanno incorporato il trattamento di tutte le più comuni fitopatologie riscontrate nel territorio considerato e per il vitigno considerato (peronospora, oidio, tignola, botrite). Nello specifico sono stati selezionati all'interno dei database i principi attivi e le dosi specifiche comunemente utilizzate negli agro farmaci utilizzati per la lotta a queste patologie. Per quanto riguarda i processi di fertilizzazione è stato ipotizzato l'utilizzo di un fertilizzante con titolo 12-12-17 (Nitrophoska Special) e l'utilizzo di altri prodotti ad azione 
Tabella 2. Fertilizzazione del terreno.

\begin{tabular}{|l|l|l|}
\hline & Caso 1 & Caso 2 \\
\hline $\mathrm{kg} / \mathrm{ha}$ & 200 & 600 \\
\hline $\mathrm{N}$ & 24 & 72 \\
\hline $\mathrm{P} 2 \mathrm{O} 5$ & 24 & 72 \\
\hline $\mathrm{K} 2 \mathrm{O}$ & 34 & 102 \\
\hline
\end{tabular}

Tabella 3. Schema e prodotti per la concimazione fogliare.

\begin{tabular}{|c|c|c|c|}
\hline $\begin{array}{c}\text { Fase } \\
\text { fenologica }\end{array}$ & Prodotto & $\begin{array}{c}\text { dose (1 o } \\
\mathrm{kg} / \mathrm{ha} \text { ) }\end{array}$ & $\begin{array}{c}\text { numero } \\
\text { applicazioni }\end{array}$ \\
\hline \multirow{3}{*}{$\begin{array}{l}\text { sviluppo } \\
\text { vegetativo }\end{array}$} & MEGAFOL & 2.5 & \multirow{3}{*}{$\begin{array}{c}2 \text { applicazioni } \\
\text { ogni } 7-10 \\
\text { giorni }\end{array}$} \\
\hline & $\begin{array}{l}\text { BREXIL } \\
\text { COMBI }\end{array}$ & 1 & \\
\hline & $\begin{array}{c}\text { PLANTAFOL } \\
30.10 .10\end{array}$ & 2.5 & \\
\hline \multirow{3}{*}{ pre-fioritura } & MEGAFOL & 2.5 & \multirow{3}{*}{1 applicazione } \\
\hline & BOROPLUS & 1 & \\
\hline & $\begin{array}{c}\text { PLANTAFOL } \\
10.54 .10 \\
\end{array}$ & 2.5 & \\
\hline \multirow{3}{*}{ allegagione } & MEGAFOL & 2.5 & \multirow{3}{*}{$\begin{array}{c}2 \text { applicazioni } \\
\text { ogni } 7-10 \\
\text { giorni }\end{array}$} \\
\hline & BOROPLUS & 1 & \\
\hline & $\begin{array}{c}\text { PLANTAFOL } \\
10.54 .10 \\
\end{array}$ & 2.5 & \\
\hline \multirow{3}{*}{$\begin{array}{l}\text { ingrossame } \\
\text { nto acino }\end{array}$} & MC CREAM & 2 & \multirow{3}{*}{$\begin{array}{c}3 \text { applicazioni } \\
\text { ogni } 7-10 \\
\text { giorni }\end{array}$} \\
\hline & BREXIL DUO & 3 & \\
\hline & $\begin{array}{c}\text { PLANTAFOL } \\
5.15 .45\end{array}$ & 3 & \\
\hline \multirow{3}{*}{ Invaiatura } & MEGAFOL & 2 & \multirow{3}{*}{$\begin{array}{c}2 \text { applicazioni } \\
\text { ogni } 7-10 \\
\text { giorni }\end{array}$} \\
\hline & SWEET & 4 & \\
\hline & $\begin{array}{c}\text { PLANTAFOL } \\
5.15 .45\end{array}$ & 3 & \\
\hline
\end{tabular}

fogliare (le cui specifiche tecniche e l'analisi di carbon footprint sono stati forniti direttamente dalla azienda produttrice Valagro SPA) in sinergia con i trattamenti fitosanitari.

Per il Caso 2 il quantitativo di NPK (con medesimo titolo) è di $600 \mathrm{~kg}$, mentre non sono previste concimazioni fogliari. In riferimento ai prodotti fogliari è stato ipotizzato un piano di utilizzo riportato nella Tabella 3.

In riferimento alle lavorazioni meccaniche è stato ipotizzato per il Caso 1 che vengano effettuati solo 2 passaggi con il trinciasarmenti (post potatura, pre raccolta) ed un passaggio con la vanga per l'interramento dei sarmenti trinciati, mentre per il Caso 2 sono previste due lavorazioni in profondità (vangatura e fresatura) e un passaggio con trinciasarmenti. Attraverso questa sperimentazione si è voluto valutare in maniera teorica il contributo dei fertilizzanti al processo produttivo dell'uva. Questa valutazione è stata effettuata attraverso l'utilizzo del software SIMAPRO 8.01. Le basi di dati prese in considerazione sono quelle di Ecoinvent 3.0 [10] per la costruzione dei processi principali inseriti nello schema di produzione. I dati primari (consumo di combustibile, lavorazioni meccaniche, principi attivi e quantitativi dei formulati fitosanitari) sono basati su
Tabella 4. Emissioni derivanti dalla fase viticola $\left(\mathrm{kgCO}_{2} \mathrm{eq} / \mathrm{ha}\right)$.

\begin{tabular}{|c|c|c|c|}
\hline \multicolumn{2}{|c|}{ Operazione colturale } & Caso 1 & Caso 2 \\
\hline \multirow{3}{*}{$\begin{array}{l}\text { Lavorazioni } \\
\text { meccaniche }\end{array}$} & Vangatura & \multicolumn{2}{|c|}{437.03} \\
\hline & Trinciatura & 388.47 & 194.23 \\
\hline & Fresatura & 0 & 410.29 \\
\hline \multirow{4}{*}{ Concimazione } & Concime NPK & 392.99 & 1183.61 \\
\hline & Concimi fogliari & 38.48 & 0 \\
\hline & Spandimento & \multicolumn{2}{|c|}{100.35} \\
\hline & Emissioni di NOx & 139.49 & 420,13 \\
\hline \multirow{2}{*}{$\begin{array}{l}\text { Trattamenti } \\
\text { fitosanitari }\end{array}$} & Agrofarmaci & \multicolumn{2}{|c|}{262.90} \\
\hline & Spandimento & \multicolumn{2}{|c|}{381.05} \\
\hline \multicolumn{2}{|c|}{ Raccolta uva } & \multicolumn{2}{|c|}{397.65} \\
\hline \multirow{3}{*}{$\begin{array}{c}\text { Totale } \\
\text { emissioni }\end{array}$} & $\begin{array}{c}\text { da combustione } \\
\text { gasolio }\end{array}$ & 1704.55 & 1920.6 \\
\hline & da agrofarmaci & 262.90 & 262.90 \\
\hline & da concimazione & 570.96 & 1603.74 \\
\hline \multicolumn{2}{|c|}{ Totale generale } & 2538.41 & 3787.24 \\
\hline
\end{tabular}

dati raccolti in precedenti studi sperimentali [33-36]. Per quanto riguarda gli impatti considerati si è fatto riferimento alla metodologia IPCC 2007 GWP 100 V1.02 [25-31]. Infatti essa risulta essere la più accurata e robusta per i fini che si propone lo studio. In riferimento alle PCR (product category rules) seguite nel corso della sperimentazione, si può dire che sebbene a livello internazionale esistano già documenti approvati e dedicati al vino [11,12], per la sperimentazione effettuata è stato seguito il protocollo sviluppato in sede OIV [30].

\section{Risultati e discussione}

Di seguito si riportano i risultati ottenuti dalla elaborazione dei dati previsti per la sperimentazione della impronta di carbonio nella fase viticola.

Come si può notare dalla tabella precedente le lavorazioni meccaniche (erpicatura, vangatura, frangizollatura, ripper, trinciatura, fresatura) sono operazioni frequenti nella realtà produttiva abruzzese e pertanto sono state inserite all'interno del nostro studio. Esse sono usualmente effettuate per fronteggiare le malerbe in campo e per permettere che gli eventuali apporti di fattori nutrizionali aggiuntivi (acqua e azoto) possano essere facilmente assorbiti dalle piante. Le lavorazioni meccaniche, a seconda della pendenza del terreno e dello sforzo a cui si sottopone il mezzo, presentano consumi di carburante più o meno elevati (circa 4-5 1/ora di gasolio per il ripper o la frangizollatura a 7-91/ora per trinciatura vangatura o fresatura). Nel contesto regionale le pratiche più diffuse sono quelle della trinciatura, della vangatura, e della fresatura. Nel nostro studio abbiamo previsto due sistemi differenti. Il primo (Caso 1) con due passaggi di trinciasarmenti ed uno di vangatrice permette di non stressare ed impoverire il soprasuolo e di apportare (attraverso l'interramento dei sarmenti) sostanza organica al terreno, limitando in questo modo la necessità di una pesante concimazione di fondo. Il secondo (Caso 2) con due lavorazioni meccaniche pesanti (vangatura e fresatura) 
ed un passaggio con trinciasarmenti. Si nota peraltro come la vangatura e la fresatura presentino consumi di gasolio notevolmente più elevati (più del doppio) rispetto alla semplice trinciatrice. Questo determina di conseguenza emissioni di $\mathrm{CO}_{2}$ doppie per questo tipo di lavorazioni. L'aspetto della concimazione è, come si può notare facilmente dalla tabella, l'elemento chiave per la valutazione delle emissioni di GHG all'interno della fase di produzione dell'uva. In questo aspetto specifico le emissioni (di GHG) relative ai fertilizzanti possono essere divise in due principali classi, quelle derivanti dalla produzione degli stessi e quelle derivanti dalla degradazione dei concimi una volta utilizzati in campo. Nel nostro caso specifico sono state calcolate entrambe (produzione e degradazione) per un concime NPK con titolo 12:12:17 (Nitrophoska special). Le emissioni prodotte dalla decomposizione del concime una volta a contatto con il terreno e con l'atmosfera variano fra valori compresi fra lo $0,5 \%$ e il $4 \%$ [10-18,23-25] dell' azoto presente nel concime e rispetto ad altri variabili legate alla composizione del terreno; tuttavia nel nostro caso è stato scelto di assumere un valore fisso di $1,98 \%$ di $\mathrm{N}_{2} \mathrm{O}$. Dalla tabella si può notare che la sola produzione del concime NPK ed il suo trasporto sul luogo di utilizzo genera emissioni che variano da 390 a $1180 \mathrm{kgCO}_{2}$ (Caso 1 e Caso 2), mentre le emissioni di GHG correlate alla decomposizione dei concimi rappresentano quasi il $30 \%$ di quelle legate alla produzione del concime. Un tale valore è determinato dal potenziale di riscaldamento globale associato alle emissioni di protossido di azoto $\left(1 \mathrm{~kg} \mathrm{~N} \mathrm{~N}_{2} \mathrm{O}=\right.$ $\left.298 \mathrm{~kg} \mathrm{CO}_{2 \mathrm{eq}}\right)$. Da questa analisi si può notare come il processo di fertilizzazione del terreno (sia con apporto di prodotti NPK standard che con un uso congiunto di prodotti fogliari) rappresenti, all'interno del bilancio globale della fase agricola, una voce importantissima (dal $30 \%$ al $45 \%$ ). Una possibile riduzione di questi valori potrebbe essere trovata con la tecnica del sovescio (semina e interramento del favino per fornire al terreno parte dell'azoto perso). Essa tuttavia viene praticata solo in pochissimi casi all'interno del territorio regionale. In rapporto alla sua importanza ed alla funzione che svolge (fissazione dell' azoto atmosferico) esso risulta fortemente sottostimato come pratica colturale. L'uso di questa tecnica permetterebbe sia apporti di azoto che di sostanza organica (50-150 kg N/ha, $10-35 \mathrm{P}_{2} \mathrm{O}_{5}, 30-120 \mathrm{~K}_{2} \mathrm{O}$ ), ed un mancato uso di fertilizzanti azotati (sia organici che di sintesi, con mancate emissioni di $\mathrm{N}_{2} \mathrm{O}$ ) e per ultimo una gestione razionale delle malerbe (che in sua presenza non crescono). I consumi energetici per ettaro per questa pratica sono da attribuire alle operazioni di semina, interramento, trinciatura e vangatura. Per un quantitativo totale di gasolio che varia da 30 a 45 1/ha ed emissioni correlate che vanno dai $130-180 \mathrm{~kg} \mathrm{CO}$ eq/ha. Questi valori sono notevolmente inferiori a quelli di una gestione convenzionale del terreno che prevede fertilizzazione (con prodotti di sintesi) e gestione meccanica delle malerbe. Tale gestione comporta consumi di gasolio simili, ai quali tuttavia occorre aggiungere le emissioni correlate con la produzione e l'utilizzo del fertilizzante.

Dalla tabella si può notare come gli agrofarmaci non rappresentino una voce molto importante all'interno del bilancio generale dei GHG. Per la sola produzione di questi composti ed il loro trasporto in azienda il contributo in termini di emissioni di $\mathrm{CO}_{2}$ eq è di circa $260 \mathrm{Kg}$. Questo valore si riferisce al solo contributo che i prodotti (valutati in circa $3 \mathrm{~kg} / \mathrm{ha}$ di prodotto per una media di 10 trattamenti) apportano al riscaldamento globale; esso non tiene conto di nessuna altra categoria di impatto ambientale alla quale probabilmente gli agrofarmaci possono contribuire (ecotossicità, piogge acide, biodiversità, ect). Ben più importante, per la voce dei trattamenti fitosanitari, appare invece il contributo dei macchinari agricoli che ammontano a circa $380 \mathrm{Kg} \mathrm{CO} \mathrm{CO}_{2} \mathrm{eq}$, essi infatti sono ripetuti numerose volte nell'arco della stagione produttiva (per le coltivazioni biologiche il numero dei trattamenti può aumentare anche del 50\%-70\%) e il dispendio di combustibile fossile per questa operazione è elevato (7/10 litri/ha per singolo trattamento) [20]. Nella analisi dei dati aggregati si può notare come la voce più importante è quella relativa alle emissioni generate dalla combustione di gasolio. In questo senso infatti un fattore da considerare è quello inerente l'efficienza energetica dei macchinari agricoli utilizzati nella fase agricola. Il parco macchine Italiano $[21,22,26]$ risulta molto vecchio per le trattrici e per gli attrezzi a loro associati (da 15 a 20 anni in media). Questo dato è significativo anche per le emissioni e per l'efficienza energetica associata ai macchinari che negli ultimi 20 anni è andata crescendo. Per quanto riguarda la raccolta dell'uva si può notare che essa, se condotta in modo meccanizzato, rappresenta una voce che può variare dal $10 \%$ al $15 \%$ circa (sul totale delle emissioni della fase agricola). I fattori che la influenzano sono ovviamente la capacità oraria dei macchinari utilizzati e le condizioni morfologiche (pendenza) del vigneto. Nel presente lavoro non sono state fatte considerazione relative al metodo biologico, in quanto esso trascende dalle finalità del presente studio e pur se molto diffuso come pratica di gestione del vigneto rappresenta ancora oggi una realtà limitata nella regione Abruzzo. Un ulteriore elemento da prendere in considerazione è quello della organizzazione della filiera. In Abruzzo sono presenti sia cantine cooperative che private. Dall'analisi della letteratura [38] si può notare che le aziende vitivinicole private sono quelle che presentano mediamente il bilancio di GHG più contenuto. Ciò può essere facilmente riscontrato nella organizzazione della gestione aziendale e nell'integrazione verticale (con la possibilità di gestire sinergicamente le fasi agricola e quella enologica). Questi due presupposti permettono una razionalizzazione di tutte le operazioni agricole (con maggiori economie di scala) ed in conclusione può essere affermato che la specializzazione della azienda vitivinicola (intesa come monocultura, spesso non presente fra le realtà che conferiscono nelle cantine cooperative) ha fra le sue principali caratteristiche una conduzione più professionale dei fondi ed in media minimizza le emissioni di GHG a livello agricolo. Le motivazioni di base sono da ricercarsi in una maggiore esperienza e professionalità nella conduzione del fondo e molto spesso nella presenza di conoscenze scientifiche di base (di carattere agronomico), congiuntamente anche ad una presenza costante sui terreni aziendali. 


\section{Conclusioni}

L'applicazione della metodologia del Carbon Footprint ai casi studio proposti risulta particolarmente interessante in quanto mette in luce aspetti della filiera viticola che fino ad oggi non sono stati interessati da studi dedicati.

Sulla base delle premesse fatte ad inizio lavoro (produttività vigneto 12 ton) si possono effettuare delle valutazioni di carattere quantitativo. Infatti sulla base dei risultati presentati in Tabella 4 si può notare come per un livello produttivo di 12 ton/ha il carico di GHG per $\mathrm{kg}$ di uva prodotto è di $0.211 \mathrm{~kg} \mathrm{CO} 2 \mathrm{eq} / \mathrm{kg}$ di uva prodotto (Caso 1), mentre è di $0.315 \mathrm{~kg} \quad \mathrm{CO}_{2 \mathrm{eq}} / \mathrm{kg}$ per il Caso 2. Questi valori sono particolarmente interessanti soprattutto se si nota che il divario di emissioni è causato principalmente dalla gestione del processo di fertilizzazione. Si può notare anche che la difesa dalle patologie influisce per valori del $10 \%-15 \%$ sul totale, mentre sono proprio le emissioni dirette ed indirette derivanti dal ciclo di fertilizzazione che appaiono di grande entità. Si ribadisce il concetto che il presente studio è stato condotto a livello teorico su uno scenario produttivo regionale (Abruzzo, vitigno Montepulciano) creato ad hoc sulla base delle esperienze degli autori. Proprio per questo motivo si ritiene opportuno precisare che prove in campo di questo tipo saranno condotte su areali produttivi di dimensioni adeguate e su un numero di campioni che possa dare rappresentatività alle varie tipologie di vigneti presenti nel nostro paese. È noto infatti che esistono altre realtà produttive che presentano rese per ettaro totalmente differenti (da 57 ton a 20-25 ton) a quella proposta e per le quali quindi i carichi medi delle emissioni riconducibili alla gestione colturale del vigneto (lavorazioni del terreno, difesa dalle patologie, concimazione, gestione aerea) sarebbero differenti a quelli dello scenario Abruzzese. Tuttavia, è proprio per questo motivo che si auspica che nel futuro possano essere condotti numerosi studi applicativi mirati alla quantificazione degli impatti ambientali generati nelle varie fasi produttive.

Da quanto emerso nella sperimentazione appare evidente che la gestione del terreno, della pianta e le pratiche colturali sono i driver fondamentali nel bilancio totale delle emissioni di GHG. L'efficienza energetica dei mezzi tecnici (trattrici e mezzi meccanici per raccolta delle olive e potatura della pianta) risultano correlati con i consumi energetici e di conseguenza con le emissioni di $\mathrm{CO}_{2}$. Tecniche colturali con un basso numero di lavorazioni meccaniche del suolo (per l'apporto di azoto) risultano di gran lunga apprezzabili per consentire la riduzione del ricorso a fertilizzanti di sintesi. In questo contesto non è stato trattato lo stoccaggio di carbonio all'interno delle strutture arboree delle piante e nel suolo. La quantificazione nelle strutture aeree della pianta è agevole tuttavia appartiene al ciclo breve della $\mathrm{CO}_{2}$ (perché inferiore ai 100 anni). Il carbonio stoccato nelle radici e quello accumulato nel terreno possono invece rientrare nella sfera del ciclo lungo della $\mathrm{CO}_{2}$. In questo ambito [39], sebbene siano parecchi gli studi a riguardo, la quantificazione del carbonio (nella struttura della piante e nel suolo) appare ancora di difficile fattibilità e sottoposta ad una elevata dose di incertezza [32-35,40,41]. Per questi motivi, a livello internazionale, sia per il vigneto (all'interno dell'OIV) che per molte altre colture, questi aspetti sono ancora in fase di definizione $[1,2]$ e vengono spesso affrontati, ma non inseriti all'interno del bilancio generale delle emissioni. In una futura applicazione pratica [17] della presente sperimentazione a contesti regionali e nazionali si procederà non tramite interviste dirette (che comunque non possono essere del tutto escluse dal metodo) ma attraverso uno studio documentale dei quaderni di campagna che potranno essere forniti alla fine della raccolta e saranno concepiti in modo da:

- rendere agevole la compilazione da parte degli imprenditori agricoli

- rrendere pratica, veloce e precisa la trasformazione dei loro contenuti in dati primari per lo studio di LCA o di CFP.

In conclusione si può affermare che la valutazione del ciclo di vita (LCA) rappresenta uno strumento potente nella valutazione e quantificazione dei carichi ambientali connessi con l'attività vitivinicola. La sua limitazione ad un solo parametro ambientale, (riscaldamento globale in questo studio) per quanto esso risulti il più attuale nel panorama globale, è un elemento limitante per la comprensione completa dei carichi ambientali connessi alla filiera del vino.

Sebbene ci siano ancora aspetti da migliorare nella fase di raccolta dei dati e nella scelta delle varie opzioni applicative della metodologia, lo studio condotto è risultato uno strumento forte ed importante per capire quale sia effettivamente il contributo delle pratiche agronomiche al bilancio generale dei gas serra. Si ringrazia particolarmente Valagro SPA per la disponibilità mostrata nel fornire tutta quanta la documentazione necessaria allo sviluppo del presente studio.

\section{Riferimenti}

[1] Aubinet, M., Grelle, A., Ibrom, A., et al.: Estimates of the annual net carbon and water exchange of forests: The EUROFLUX methodology. Adv. Ecol. Res. 30: 113-175, 2000

[2] Baldocchi, D.D., Hicks, B.B., Meyers, T.P.: Measuring biosphere-atmosphere exchange of biologically related gases with micrometeorological methods. $t$ Ecology 69: 1331-1340, 1988

[3] Benedetto, G. (2013). The environmental impact of a Sardinian wine by partial Life Cycle Assessment. Wine Economics and Policy, 2, 33-41

[4] Bosco, S., Di Bene, C., Galli, M., Remorini, D., Massai, R., \& Bonari, E. (2011). Greenhouse gas emissions in the agricultural phase of wine production in the Maremma rural district in Tuscany, Italy. Italian Journal of Agronomy, 6(15), 93-100

[5] Bosco, S., Di Bene, C., Galli, M., Remorini, D., Massai, R., \& Bonari, E. (2013). Soil organic matter accounting in the carbon footprint analysis of the wine chain. The International Journal of Life Cycle Assessment, 18(5), 973-989

[6] Cappelletti G.M., Cichelli A., Michalopoulos G., Nicoletti G.M., Polo Palomino J.A., Pattara C., Saouter E., Russo C., Tuomisto H.. Defining the 
Product Environmental Footprint Category Rules (PEFCR) for the Olive Oil and Table Olive Sectors. Strumenti per la valutazione della sostenibilità di prodotti/processi: Environmental and Social LCA, Life Cycle Costing, Environmental Footprint, Carbon \& Water Footprint, Emergy, MFA A cura di: CTS Ecomondo, Rete Italiana LCA, Divisione di Chimica dell'Ambiente e dei Beni culturali, ENEA, Università di Bologna

[7] Cerutti, A.K., Beccaro, G.L., Bruun, S., Bosco, S., Donno, D., Notarnicola, B., Bounous, G. (2013). LCA application in the fruit sector: state of the art and recommendations for environmental declarations of fruit products. (2013) Journal of Cleaner Production, 73, 125-135. http://dx. doi.org/10.1016/j.jclepro.2013.09.017

[8] Cholette, S., \& Venkat, K. (2009). The energy and carbon intensity of wine distribution: A study of logistical options for delivering wine to consumers. Journal of Cleaner Production, 17, 1401-141

[9] Cichelli A.; Procida G.; Di Martino M.; Pattara C. 2011. Analisi dei composti volatili di vini conservati in legno e con materiali legnosi, XXV Congresso Nazionale di Scienze Merceologiche. Trieste-Udine. 26-28 settembre. FORUM Edizioni. pp.18-22. ISBN 9788884207050

[10] Ecoinvent, 2010. Ecoinvent Data V2.2. Swiss Centre for Life Cycle Inventories.FIVS (2008)

[11] EPD. (2006). Product Category Rules (PCR) for preparing an environmental product declara-tion (EPD) for Packaged Sparkling red, white and rosé wines. PCR 2006:03. The International $\mathrm{EPD}^{\circledR}$ System, Version 1.0

[12] EPD. (2013). Product Category Rules (PCR) for preparing an environmental product declara-tion (EPD) for UN CPC 24212- Wine of fresh Grapes, except sparkling wine; Grape must. PCR 2010:02. The International EPD ${ }^{\circledR}$ System, Version 1.03

[13] European Commission (2013). Communication from the commission to the European Parliament and the council Building the Single Market for Green Products Facilitating better information on the environmental performance of products and organisations, EUR-Lex 52013DC0196 - EN. http://eur-lex.europa. eu/legal content/IT/TXT/PDF/?uri=CELEX : 52013DC0196\&from $=$ EN

[14] European Commission (2013). Attitudes of europeans towards building the single market for green products, Flash Eurobarometer 367, July 2013. http://ec.europa.eu/public_opinion/flash/ fl_367_en.pdf

[15] European Commission (2013). 2013/179/EU: Commission Recommendation of 9 April 2013 on the use of common methods to measure and communicate the life cycle environmental performance of products and organisations. http:// eur-lex. europa.eu/legal-content/EN/TXT/? uri $=$ CELEX : $32013 \mathrm{H} 0179$

[16] European Commission (2011). Analysis of Existing Environmental Footprint Methodologies for Products and Organizations: Recommendations, Rationale, and Alignment. Deliverable 1 to the Administrative Arrangement between DG Environment and Joint Research Centre No. N 070307/2009/552517, including Amendment No 1 from December 2010. http://ec.europa.eu/environment/eussd/pdf /Deliverable.pdf

[17] Fedele A., Mazzi A., Niero M., Zuliani F., Scipioni A., (2014).Can the life cycle assessement methodology be adopted to support single farm on its environmental impact forecast evaluation between conventional and organic production? An Italian case study. Journal of Cleaner Production, 69, 49-59

[18] Greenhouse Gas Protocol - Product Life Cycle Accounting and Reporting Standard, 2011

[19] Hayes, P., \& Battaglene, T. (2006). Regulatory response to climate change. Le Bulletin de L' OIV. Organisation Internationale de La Vigne et du Vigne, 79, 697-708

[20] International Energy Agency, (2013). $\mathrm{CO}_{2}$ Emissions From Fuel Combustion Highlights 2013. At http: // www .iea.org/publications/freepublications /publication/co2-emissions-from-fuel-com bustion-highlights-2013-.html?direct $=1$ last access 22 october 2014

[21] INEA1, (2013). La meccanizzazione agricola in Italia. Aspetti tecnici, economici, ambientali e sociali. CSR Centro Stampa e Riproduzione, ISBN 978-88-8145-270-5

[22] INEA2, (2013), Rapporto sullo stato dell'agricoltura. 2013, Pubblicazioni congiunturali e ricerche macroeconomiche. Rapporti. INEA 2013

[23] IPCC. 1997, Revised 1996 IPCC Guidelines for National Greenhouse Inventories. Prepared by the IPCC/OECD/IEA, Paris, France. J.T., Houghton, Melra Fllho L.G., Um B., Trdanton K.. Mamaty I. Bonduki Y., Griggs D.J. and Callander B.A. (Eds)

[24] Intergovernmental Panel on Climate Change (2006). Guidelines for National Greenhouse Gas Inventories.

[25] Intergovernmental Panel on Climate Change, 2013. Climate Change 2013: The Physical Science Basis. http://www.ipcc.ch/report/ar5/wg1/ (last access October 22/2014)

[26] ISTAT, (2013). Atti del $6^{\circ}$ Censimento Generale dell'Agricoltura. 2013. ISBN: 978-88-458-1779-3 (elettronico)

[27] Lal R., (2004). Soil carbon sequestration to mitigate climate change. Geodema, 123, 1-22

[28] F. Murmura, C. Pattara, A. Cichelli, Technical and economic evaluation concerning the different employment of marc. International Journal of Food, Agriculture \& Environment (JFAE) 2009 Volume 7, Issue 3\&4. ISSN 1459-0255 pages 925-929

[29] Notarnicola B., Salomone R., Petti L., Renzulli P.A., Roma R., Cerutti A. K. Life Cycle Assessment in the agri-food sector. International practices, Italian experiences, methodological issues and definition of practical guidelines Springer in press

[30] OIV. (2011). General principles of the OIV greenhouse gas accounting protocol for the vine and wine sector. Resolution OIV-CST 431-2011 
[31] PAS 2050:2011, Specification for the assessment of the life cycle greenhouse gas emissions of goods and services

[32] Pattara C., Cappelletti G.M., Cichelli A., (2010) Recovery and use of olive stones: commodity, environmental and economic assessment, Renewable and Sustainable Energy Rewiews, 14 (5), 1484-1489

[33] Pattara, C., Cichelli, A., Civitarese, C., \& Di Martino, M. (2012a). A comparison of carbon footprints in wine production: The case of two cooperative wineries in central Italy. Bulletin de l'OIV, 85 (977978-979), 307-316

[34] Pattara, C., Raggi, A., \& Cichelli, A. (2012b). Life Cycle Assessment and carbon footprint in the wine supply-chain. Environmental Management, 49, 1247-1258

[35] Pattara C., Civitarese C., Salomone R., 2014. La valutazione del carbon footprint nella filiera olivicolo-elaiotecnica nel centro Italia. Il caso studio dell' Abruzzo. Atti del XXVI Congresso Nazionale di Scienze Merceologiche, Pisa 13-15 Febbraio 2014. Pag 532-542. ISBN 978-1-291-74318-0

[36] Pattara C., Raggi A., Cichelli A., Life Cycle Assessment and Carbon Footprint in the wine supplychain, Proc. LCA FOOD 2010, 7th Intl Conf. Life Cycle Assessment in the Agri-Food Sector, Bari, Italy, 22-24 September 2010, pp.141-146. ISBN 978-88-88793-29-0

[37] Pelletier, N., Ibarburu, M., \& Xin, H. (2013). A carbon footprint analysis of egg production and processing supply chains in the Midwestern United States. Journal of Cleaner Production, 54, 108-114

[38] Petti, L., F. Ardente, S. Bosco., C. De Camillis, P. Masotti, C. Pattara, A. Raggi, G. Tassielli (2010), "State of the art of Life Cycle Assessment (LCA) in the wine industry", Proc. LCA FOOD 2010, 7th Intl Conf. Life Cycle Assessment in the AgriFood Sector, Bari, Italy, 22-24 September 2010, pp. 493-498. ISBN 978-88-88793-29-0

[39] Prentice, I.C., 2001. The carbon cycle and the atmospheric carbon dioxide. Climate Change 2001: The Scientific Basis. Intergovernmental Panel on Climate Change. Cambridge Univ. Press, UK, pp. 183- 237

[40] Salomone, R., Cappelletti, G.M., Ioppolo, G., Mistretta, M., Nicoletti, G., Notarnicola, B., Olivieri, G., Pattara, C., Russo, C., Scimia, E., 2010. Italian experiences in life cycle assessment of olive oil: a survey and critical review. In: VII International Conference on Life Cycle Assessment in the AgriFood Sector, vol. 2. Conference Proceedings, Bari, 22-24 September, pp. 265-270

[41] Salomone R., Cappelletti G.M., Malandrino O., Mistretta M., Neri E., Nicoletti G.M., Notarnicola B., Pattara C., Russo C. and Saija G. (2015). Life Cycle Assessment in the Olive Oil Sector, chapter in Life Cycle Assessment in the Agri-food Sector (2015). Notarnicola B

[42] Xiloyannis C., Palese A.M., Egidio Lardo E., Fiore A., Montanaro G., (2014). Protocollo di gestione dell'oliveto finalizzato all'aumento del carbonio nel suolo e nelle strutture vegetali 